\title{
The functional significance of newly born neurons integrated into olfactory bulb circuits
}

\author{
Masayuki Sakamoto ${ }^{1,2+}$, Ryoichiro Kageyama ${ }^{1,3,4 *}$ and Itaru Imayoshi ${ }^{1,3,5,6 *}$ \\ 1 Institute for Virus Research, Kyoto University, Kyoto, Japan \\ ${ }^{2}$ Kyoto University Graduate School of Biostudies, Kyoto, Japan \\ ${ }^{3}$ World Premier International Research Initiative-Institute for Integrated Cell-Material Sciences, Kyoto University, Kyoto, Japan \\ 4 Japan Science and Technology Agency, Core Research for Evolutional Science and Technology, Kyoto, Japan \\ ${ }^{5}$ The Hakubi Center, Kyoto University, Kyoto, Japan \\ ${ }^{6}$ Japan Science and Technology Agency, Precursory Research for Embryonic Science and Technology, Kyoto, Japan
}

\section{Edited by:}

Luca Bonfanti, University of Turin, Italy

\section{Reviewed by:}

Francesca Ciccolini, University of Heidelberg, Germany

Liliana Bernardino, University of

Beira Interior, Portugal

${ }^{*}$ Correspondence:

Ryoichiro Kageyama, Institute for

Virus Research, Kyoto University,

Shogoin-Kawahara, Sakyo-ku,

Kyoto 606-8507, Japan

e-mail: rkageyam@

virus.kyoto-u.ac.jp;

Itaru Imayoshi, The Hakubi Center, Institute for Virus Research, Kyoto

University, Shogoin-Kawahara,

Sakyo-ku, Kyoto 606-8507, Japan

e-mail: iimayosh@virus.kyoto-u.ac.jp

${ }^{\dagger}$ Present address:

Masayuki Sakamoto, Department of

Biological Sciences, Columbia

University, New York, USA
The olfactory bulb (OB) is the first central processing center for olfactory information connecting with higher areas in the brain, and this neuronal circuitry mediates a variety of odor-evoked behavioral responses. In the adult mammalian brain, continuous neurogenesis occurs in two restricted regions, the subventricular zone (SVZ) of the lateral ventricle and the hippocampal dentate gyrus. New neurons born in the SVZ migrate through the rostral migratory stream and are integrated into the neuronal circuits of the $\mathrm{OB}$ throughout life. The significance of this continuous supply of new neurons in the $\mathrm{OB}$ has been implicated in plasticity and memory regulation. Two decades of huge investigation in adult neurogenesis revealed the biological importance of integration of new neurons into the olfactory circuits. In this review, we highlight the recent findings about the physiological functions of newly generated neurons in rodent OB circuits and then discuss the contribution of neurogenesis in the brain function. Finally, we introduce cutting edge technologies to monitor and manipulate the activity of new neurons.

Keywords: neurogenesis, main olfactory bulb, accessory olfactory bulb, granule cell, periglomerular cell, lateral inhibition, behavior, neural stem cell

\section{INTRODUCTION}

It was believed that the adult mammalian brain is incapable of producing new neurons. The prominent histologist Cajal proclaimed "Once the development was ended, the founts of growth and regeneration of the axons and dendrites dried up irrevocably. In the adult centers, the nerve paths are something fixed, ended, and immutable. Everything may die, nothing may be regenerated." (Ramon y Cajal, 1928). In 1960's, Altman and his colleague's pioneering study provided the first anatomical evidence of neurogenesis in the postnatal hippocampal region using a $\left[\mathrm{H}^{3}\right]$-thymidine incorporation labeling (Altman and Das, 1965). These $\left[\mathrm{H}^{3}\right]$-thymidine-labeling cells had neuronal morphology (Kaplan and Hinds, 1977). However, these findings were not accepted by Cajal's neuron doctrine that no new neurons are born in the adult brain. In 1980's, adult neurogenesis identified in songbird's brain was found to play a role in song learning (Goldman and Nottebohm, 1983). In 1990's, neural stem/progenitor cells were isolated from adult rodent brain, and adult neurogenesis was discovered in human hippocampus (Reynolds and Weiss, 1992; Eriksson et al., 1998). Since the discovery, adult neurogenesis has now become a wellaccepted phenomenon including humans (Sanai et al., 2011;
Bergmann et al., 2012; Spalding et al., 2013; Ernst et al., 2014).

In rodents, adult neurogenesis mainly occurs in two brain regions, the subventricular zone (SVZ) of the lateral ventricles and the subgranular zone (SGZ) of the hippocampal dentate gyrus (DG) (Kriegstein and Alvarez-Buylla, 2009; Suh et al., 2009; Aimone et al., 2011; Fuentealba et al., 2012). Adult neural stem/progenitor cells are regulated by many genes and signaling pathways (Kriegstein and Alvarez-Buylla, 2009; Suh et al., 2009). Neurons born in the SGZ migrate into the granule cell layer (GCL) and become granule cells of the DG, while neurons born in the SVZ migrate into the olfactory bulb (OB) through the rostral migratory stream (RMS), the pathway leading to the $\mathrm{OB}$, and become local interneurons, granule cells (GCs) and periglomerular cells (PGCs) (Lledo et al., 2006; Ming and Song, 2011; Lepousez et al., 2013).

The olfactory system, which senses and processes odor information, is one of the oldest and important parts of the brain. Odor information is transferred to local neural circuits in the OB, and then conveyed to various regions of the olfactory cortex via principal neurons (mitral and tufted cells, hereafter referred to these neurons as $\mathrm{M} / \mathrm{T}$ cells). Unlike most other central nervous 
system areas, GABAergic inhibitory interneurons greatly outnumber principal neurons, suggesting that odor representations in the $\mathrm{OB}$ are shaped by local inhibitory circuits (Yokoi et al., 1995; Isaacson and Strowbridge, 1998; Egger and Urban, 2006). Furthermore, although most neurons comprising the mammalian central nervous system are produced during embryonic development, a large proportion of these interneurons in the OB are generated and continuously renewed throughout life. Why do such continuous neuronal addition and replacement occur in the OB? Two decades of huge investigation revealed the biological importance of integration of new neurons into the olfactory circuits (Lledo et al., 2006; Kelsch et al., 2010; Ming and Song, 2011; Lepousez et al., 2013).

In this review, we highlight recent findings about physiological features of new neurons in rodent $\mathrm{OB}$ circuits and then describe the role of new neurons in olfaction-associated behaviors. Finally, we introduce optical techniques to monitor and manipulate the activity of new neurons.

\section{NEURONAL CIRCUIT OF THE OLFACTORY SYSTEM}

The $\mathrm{OB}$ is the first relay station in the olfactory system that can process odor information (Figure 1). Odor information is detected by olfactory sensory neurons (OSNs). OSNs expressing the same odorant receptors project and converge their axons into the same glomeruli (Mori and Sakano, 2011). OSNs form excitatory synapses on primary dendrites of M/T cells. M/T cells project their axons to the olfactory cortex to covey odor information to higher brain areas in the forebrain. Mitral cells project their axons to nearly all areas of the olfactory cortex with a dispersed manner, while tufted cells target densely only to the anterior regions of the olfactory cortex (Ghosh et al., 2011; Miyamichi et al., 2011; Sosulski et al., 2011; Igarashi et al., 2012).

Synaptic connections in the external plexiform layer (EPL) of the $\mathrm{OB}$ are dominated by dendrodendritic reciprocal synapses between lateral dendrites of $\mathrm{M} / \mathrm{T}$ cells and GCs, the latter being most numerous type of inhibitory interneurons in the OB. Unlike the neocortex, GABAergic inhibitory interneurons in the OB grealy outnumber principal neurons by $50-100: 1$ (Isaacson and Strowbridge, 1998; Egger and Urban, 2006). GCs form dendrodendritic synapses with $\mathrm{M} / \mathrm{T}$ cells. In a dendrodendritic reciprocal synapse, both sides of the synapse are dendrites. M/T to GC is a glutamatergic excitatory synapse, while $\mathrm{GC}$ to $\mathrm{M} / \mathrm{T}$ is a GABAergic inhibitory synapse (Figure $\mathbf{1}$ ). This large number of inhibitory synapses onto $\mathrm{M} / \mathrm{T}$ cells may enable inhibitory circuits to refine odor representations. (Isaacson and Strowbridge, 1998; Egger and Urban, 2006; Lepousez and Lledo, 2013).

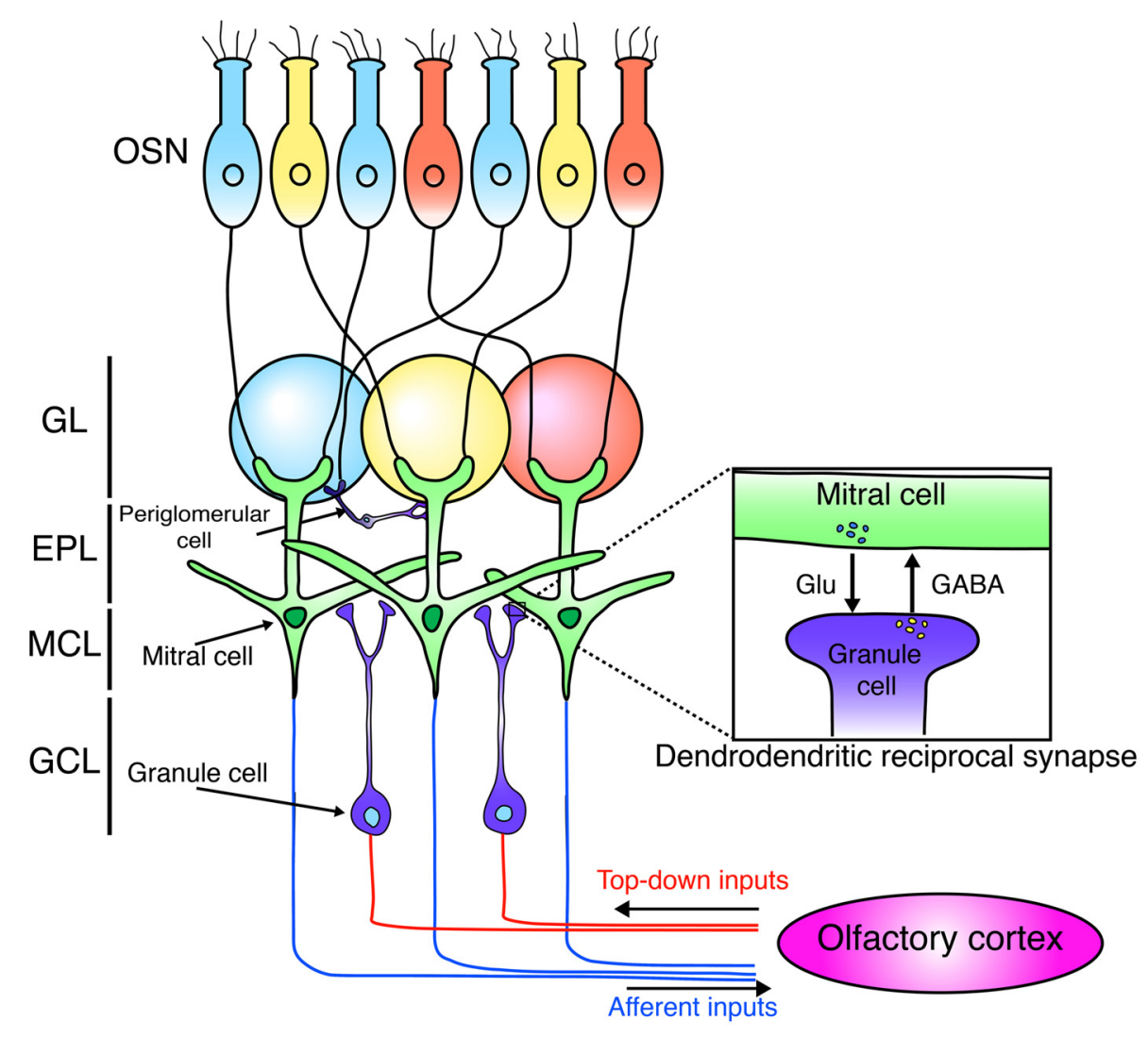

FIGURE 1 | Neural circuit of the olfactory bulb. Schematic diagram of the neuronal circuit of the olfactory bulb. OSNs expressing the same odorant receptors (blue, yellow, red) project and converge their axons into the same glomeruli. OSNs form excitatory synapses with mitral cells. Mitral cells project their axons to the olfactory cortex. Mitral cells form dendrodendritic synapses with granule cells. Granule cells receive centrifugal glutamatergic inputs from the olfactory cortex. OSN, olfactory sensory neuron; GL, glomerular layer; EPL, external plexiform layer; $\mathrm{MCL}$, mitral cell layer; $\mathrm{GCL}$, granule cell layer. 
PGCs are another type of major GABAergic interneurons in the $\mathrm{OB}$, and modulate the neural circuit in the glomerulus, consisting of terminals of the olfactory nerve and the dendrites of M/T. PGCs are subdivided into at least three subtypes based on immunoreactivity to calretinin (CalR), calbindin-28K (CalB), and tyrosine hydroxylase (TH) (Kosaka et al., 1998; Pressler and Strowbridge, 2006; Eyre et al., 2008, 2009; Kosaka and Kosaka, 2011). In mice, all three PGC subtypes seem to be GABAexpressing inhibitory neurons, but the definite functional roles of each PGC subtype in odor processing have not been well determined.

In addition to GCs and PGCs, numerous types of GABAergic interneurons have been identified in the OB (Pressler and Strowbridge, 2006; Batista-Brito et al., 2008; Eyre et al., 2008, 2009; Kosaka and Kosaka, 2011; Huang et al., 2013; Kato et al., 2013; Miyamichi et al., 2013), including deep short-axon cells, Blanes cells, and EPL interneurons. Although lineage and turnover analysis of these $\mathrm{OB}$ interneuronal populations has just been started (Batista-Brito et al., 2008; Bartolini et al., 2013), dynamic turnover of these interneurons by postnatal/adult neurogenesis may also contribute to the reorganization of $\mathrm{OB}$ circuitry.

\section{UNIOUE FEATURES OF NEWLY GENERATED NEURONS}

While M/T cells are generated only at an embryonic stage, GCs and PGCs are generated throughout life (Imayoshi et al., 2008; Imamura et al., 2011; Sakamoto et al., 2014). Long-term genetic labeling analysis revealed that the majority of GCs are replaced by newly generated neurons during adult life (Imayoshi et al., 2008). Newly generated GCs are preferentially located in a deep region, while pre-existing GCs are located in a superficial region in the GCL of the OB (Lemasson et al., 2005; Imayoshi et al., 2008; Sakamoto et al., 2014). Interestingly, it has been shown that outer/superficial GCs, whose dendrites preferentially target the superficial lamina of the EPL, establish synapses with tufted cells, whereas deep GCs mainly contact the dendrites of mitral cells in the deep lamina of the EPL (Mori et al., 1983; Orona et al., 1983; Shepherd and Greer, 2004; Imamura et al., 2006). Therefore, one attractive hypotheis is that these two GC subpopulations fundamentally modulate distinct neural circuits. This implies that the activity of tufted cells is under the preferential control of embryonic-born GCs (static, superficial layers), while postnatal-born GCs (turnover, deep layers) provide an inhibitory drive to both mitral and tufted cells.

In rodents, although numerous new neurons reach the $\mathrm{OB}$ each day (roughly one percent of the total OB GCs), only half of them are integrated into pre-existing neural circuits. The remains of them are eliminated by apoptosis during their maturation (Lledo et al., 2006). This "survival or death" depends on olfactory sensory experience. Sensory deprivation triggers a decrease in new GC survival, whereas olfactory enrichment and learning boost the survival of these neurons (Petreanu and Alvarez-Buylla, 2002; Rochefort et al., 2002). Interestingly, day 14 to 28 after the generation is a critical period of newly born GCs when their survival is influenced by sensory experience (Yamaguchi and Mori, 2005). This time window overlaps with the period when newly generated neurons make synapses with pre-existing neurons, suggesting that synaptic inputs play a crucial role in the selection of adult born GCs (Kelsch et al., 2008; Yokoyama et al., 2011). Although the number of PGCs is one order smaller than that of GCs, new PGCs are also continuously produced throughout life (Ninkovic et al., 2007; Sakamoto et al., 2014). Like GCs, the survival of newly born PGCs is regulated in an activity-dependent manner. Sensory deprivation triggers a decrease in new PGCs' survival, whereas olfactory enrichment and learning boost the survival of adult generated PGCs (Rochefort et al., 2002; Alonso et al., 2006; Adam and Mizrahi, 2011; Sawada et al., 2011; Livneh and Mizrahi, 2012). A recent work also reported the generation of some glutamatergic short-axon cells at a very low proportion (Brill et al., 2009).

One recent elegant study provided direct evidence of the involvement of adult-born PGCs in olfactory sensory processing (Livneh et al., 2014). By using two-photon-targeted patch recordings, they showed that adult-born PGCs indeed respond to odor input. Interestingly, young adult-born neurons (2-4 weeks of age) have broader odor response profile than that of matured resident PGCs. Furthermore, sensory enrichment during developmental periods of adult-born neurons sharpens their odor response selectivity after maturation. These results indicated that continuous supply of these sensitive adult-born neurons into the olfactory circuit provides it with a mechanism of long-lasting plasticity (Livneh et al., 2014).

The OB receives input not only from OSNs but also from the olfactory cortex (Figure 1). This top-down input targets preferentially to the GCL and is important to shape the activity of M/T neurons (Manabe et al., 2011; Boyd et al., 2012; Markopoulos et al., 2012). In addition, recent studies showed that this cortical feedback is necessary for odor discrimination and food-intake (Nunez-Parra et al., 2013; Soria-Gomez et al., 2014). Furthermore, several studies showed the connectivity of newly generated neurons using monosynaptic rabies virus-based tracing system and revealed that newborn neurons in the OB receive glutamatergic inputs from neurons in the olfactory cortex (Arenkiel et al., 2011; Deshpande et al., 2013). Interestingly, new neurons exhibit more synaptic plasticity from centrifugal inputs than mature neurons do (Nissant et al., 2009). Furthermore, topdown inputs on the proximal dendrites of GCs also contribute to the survival/death of new neurons (Yamaguchi et al., 2013). Therefore, these observations imply that top-down glutamatergic input from the olfactory cortex to new GCs has a critical role in generating high plasticity in $\mathrm{OB}$ cirucuits.

Adult neurogenesis occurs in human brain as well as in rodents. Radiocarbon dating technologies revealed that adult neurogenesis in the $\mathrm{OB}$ is extremely limited though hippocampal neurogenesis occurs at a steady rate (Bergmann et al., 2012; Spalding et al., 2013). Surprisingly, new neurons born SVZ/lateral ventricles migrate and differentiate into striatum interneurons (Ernst et al., 2014). Furthermore, striatum neurogenesis is reduced in patients with Huntington's diseases. These results indicate that adult neurogenesis in humans has a unique pattern, and that these neurons derived from SVZ/lateral ventricles might be involved in brain functions such as cognition and motor coordination. 


\section{THE ROLES OF NEWLY GENERATED NEURONS FOR OLFACTION-RELATED BEHAVIORS}

While the functional significance of continuous neurogenesis in hippocampus has been extensively studied (Deng et al., 2010; Aimone et al., 2011), the role of newly generated neurons in olfaction-related behaviors remains elusive. As mentioned above, newly born neurons form dendrodendritic synapses with $\mathrm{M} / \mathrm{T}$ cells and control the activity of M/T cells to shape odor representation. Genetic ablation of newly born neurons in the OB impairs the structure and neural circuits in the OB (Imayoshi et al., 2008; Sakamoto et al., 2011). It was reported that newly generated GCs exhibit long-term synaptic plasticity, and that this ability is gradually lost as these neurons become mature, indicating that newly born GCs play a more important role in plastic change than mature GCs (Nissant et al., 2009). Importantly, electrophysiological recording revealed that ablation of adult born neurons impairs recurrent and lateral dendrodendritic inhibition of $\mathrm{M} / \mathrm{T}$ cells and reduces the frequency of the induced gamma oscillations in the OB (Breton-Provencher et al., 2009). Furthermore, the survival of newly generated neurons is regulated by sensory experience (Yamaguchi and Mori, 2005; Lledo et al., 2006; Yokoyama et al., 2011). Together, these findings suggest that neurogenesis has a key role in olfaction-related plastic activities in the OB.

To understand the functional role of neurogenesis in the $\mathrm{OB}$, various behavioral analyses have been applied. To address this question, various methodologies for inhibiting neurogenesis have been used: pharmacological, irradiation, and genetic targeting (Gheusi et al., 2000; Kim et al., 2007; Bath et al., 2008; Imayoshi et al., 2008; Breton-Provencher et al., 2009; Lazarini et al., 2009; Sultan et al., 2010; Sakamoto et al., 2011). Conversely, an apoptotic inhibitor was used to suppress cell death of newly born neurons (Mouret et al., 2009; Sultan et al., 2011).

One of the simplest behavioral tests for olfaction is to check spontaneous odor exploration toward a novel odor without any rewards (Figure 2A). In this task, the ability of odor discrimination can be assessed by repeated presentations of the same odor (habituation) followed by the presentation of a novel odor (dishabituation). The sniffing time decreases during habituation sessions, but then increases when the odor is recognized as a new one in a dishabituation session. This can be applied to evaluate the odor detection threshold by comparing sniffing times between different concentrations. This test can also assess the short-term olfactory memory by changing time interval between sessions. Blockade of neurogenesis by infusion of an antimitotic drug impairs the ability of odor detection and short-term memory $(60 \mathrm{~min})$, suggesting that new neurons are involved in odor detection and processing odor memory (Breton-Provencher et al., 2009). In addition, gene deficient mice, which result in a decrease of new neurons in the $\mathrm{OB}$, could not discriminate between dissimilar odors (Gheusi et al., 2000; Bath et al., 2008). However, not all findings have supported this result. Mice treated with $\gamma$ ray irradiation to block neurogenesis showed normal sensitivity (Lazarini et al., 2009), suggesting that spontaneous odor discrimination is not affected in these mice. Similar results were obtained from other studies (Kim et al., 2007; Imayoshi et al., 2008; Sakamoto et al., 2011). The discrepancies between these findings may be due to different ablation methods. Regarding the target

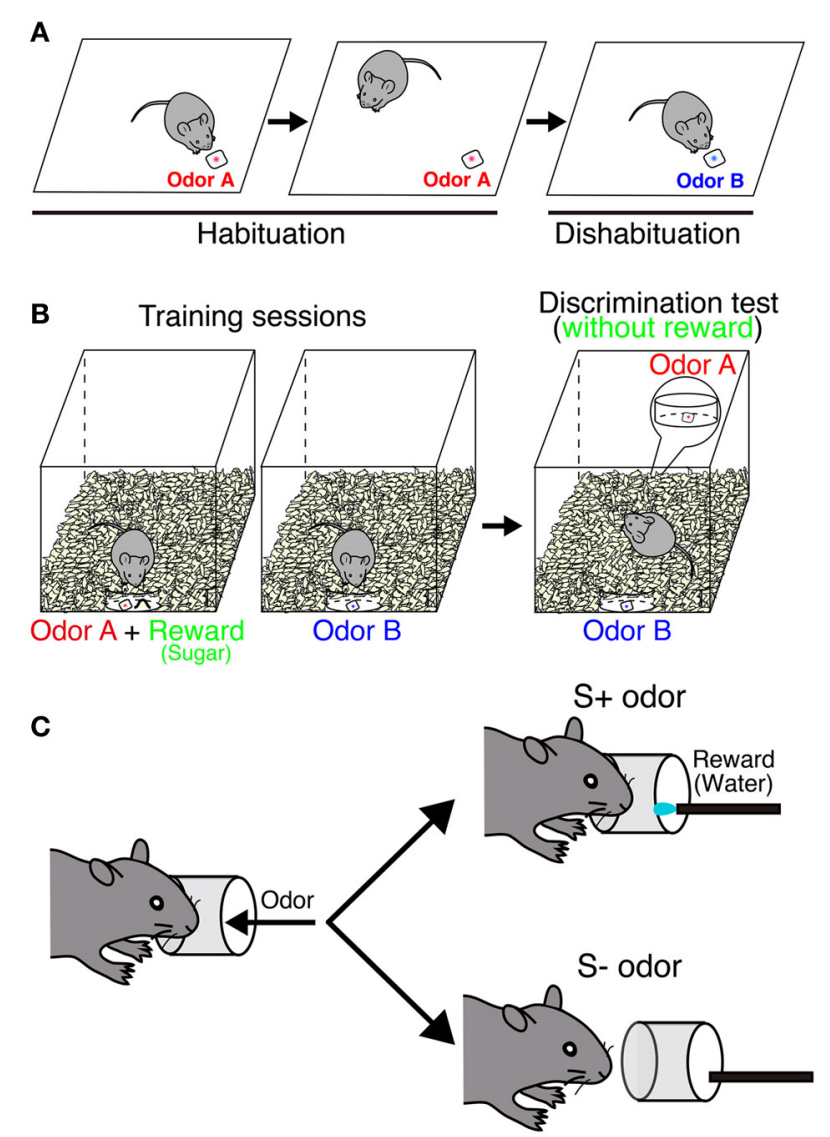

FIGURE 2 | Behavioral paradigm of odor discrimination test. (A) Habituation-dishabituation test. In habituation sessions, odor A (red) is presented repeatedly. A mouse is habituated to this odor, and sniffing time is getting decreased. In a dishabituation session, sniffing time increases when the mouse can recognize a novel odor B (blue). (B) Odor-reward association test. During the training, a mouse is associated with one of the odors with reward (sugar). In the test, both odors are placed under the bedding separately without reward. Digging time of each odorant is measured to judge whether the mouse can discriminate between the two odors. In an odor memory retention test, mice are exposed to the same test without further training. (C) Go/no-go olfactory conditioning test. A mouse is associated with one of the odors with reward (water). Each trial is counted as correct if the mouse licks continuously upon presentation of a rewarded $(\mathrm{S}+)$ odor or does not lick continuously with a non-rewarded (S-) odor.

specificity, blocking adult neurogenesis by using conventional knock in/out mice is far from specific.

Odor-reward association learning is another major paradigm that has been used to evaluate the function of neurogenesis in the OB (Figures 2B,C). This test can evaluate the capacity to associate an odor with reward (food or water) and the ability of odor discrimination and memory retention. Genetically ablated mice can acquire the odor-associated memory and maintain it for at least 2 months (Imayoshi et al., 2008; Sakamoto et al., 2011). Similar results were obtained from other animal models (Schellinck et al., 2004; Breton-Provencher et al., 2009). These results indicate that continuous neurogenesis is not required for simple discrimination between similar odors or retention of 
odor-associated memory. Pre-existing granule neurons born in embryonic and neonatal stages may compensate for these functions in the absence of new neurons. However, conflicting results were also reported following odor-reward association learning. Although mice showed normal odor discrimination ability, their long-term odor-associated memory retention was impaired by irradiation- or drug-induced inhibition of neurogenesis (Lazarini et al., 2009; Sultan et al., 2010). This discrepancy likely results from the difference in experimental paradigms. In addition, parameters to evaluate odor memory are totally different depending on behavior tests [digging time (seconds) or correct decision (\%)].

These discrepancies may result from the target specificity for blockade of neurogenesis. Strictly speaking, these past methods did not specifically target new neurons in the OB. Neurons in other cortical regions might be affected to some extent. Therefore, there is a need to generate more sophisticated model that can target only newly generated neurons in the OB. One possibility to increase the target specificity is an intersectional strategy with dual site-specific recombinases (Cre and Flp) (Imayoshi et al., 2011; Huang and Zeng, 2013). Most of new neurons in the OB are GABAergic inhibitory neurons, while new neurons in the DG are glutamatergic excitatory neurons. Based on their different transmitter characteristics, new neurons in the OB and DG can be separately targeted. Recently, our group has developed new transgenic mice in which new neurons in the OB and DG can be separately targeted, and found that continuous neurogenesis is important for flexible olfactory associative learning and memory (Sakamoto et al., 2014). Continuous supply of new neurons in the $\mathrm{OB}$ is important to rewrite aquired odor memory and modify the value of odor-associated memory.

Adult neurogenesis in the hippocampus is required for pattern separation (Clelland et al., 2009; Sahay et al., 2011a,b; Nakashiba et al., 2012). It seems that odor enrichment improves a recruitment of newly born neurons and the olfactory discrimination ability. Blockade of neurogenesis with AraC impaired the improvement of odor discrimination, suggesting that neurogenesis is required for perceptual learning (Moreno et al., 2009). This result implies that neurogenesis in the $\mathrm{OB}$ also contributes to pattern separation. It would be interesting to examine the relationship between OB neurogenesis and pattern separation (Sahay et al., 2011b).

\section{OB NEUROGENESIS IN INNATE BEHAVIORS}

Adult neurogenesis is physiologically linked to reproductive behaviors, suggesting that continuous neurogenesis plays a pivotal role in pheromone-associated behaviors (Shingo et al., 2003; Mak et al., 2007; Mak and Weiss, 2010; Nunez-Parra et al., 2011). Pregnancy and lactation increase the number of both new GCs and PGCs (Shingo et al., 2003). Around gestation day 7, the proliferation reaches a peak in the SVZ/lateral ventricles. After the delivery, the number of new neurons integrated into $\mathrm{OB}$ circuits increases, and their dendritic spines exhibit stable features (Shingo et al., 2003; Kopel et al., 2012). These phenomena during early pregnancy and parenting might be important for finetuning of olfactory response to mating partners and pups. This induction of neurogenesis is mediated by prolactin (Shingo et al.,
2003; Larsen and Grattan, 2010). Reducing the prolactin levels decreases neurogenesis in the SVZ/lateral ventricles and impairs maternal behaviors (Larsen and Grattan, 2010). Neurogenesis in females is also induced by pheromones of dominant males (but not other males) and is important for sexual behaviors (Mak et al., 2007; Oboti et al., 2009, 2011). Relevant increase of new neurons also occurs in male mice when they interact with their postnatal offspring (Mak and Weiss, 2010). This increase of neurogenesis mediated by prolactin appears to depend on the odor of their offspring and is involved in offspring recognition (Mak and Weiss, 2010).

These results indicate that $\mathrm{OB}$ neurogenesis is really related to sexual and maternal behaviors, suggesting that neurogenesis plays an important role in such pheromone-associated innatelyprogrammed behaviors. Genetic inhibition of adult neurogenesis revealed that new neurons are essential for mating and maternal behaviors (Sakamoto et al., 2011). Blocking neurogenesis by injecting antimitotic drugs also impairs mating behaviors (Oboti et al., 2011). Pregnancy block (Bruce effect) is a well-known phenomenon; females terminate their pregnancy when they are exposed to the scent of unfamiliar males (Bruce, 1959). Although the detailed mechanism of this pregnancy block remains to be determined, it was shown that the pregnancy failure rate is highly increased by the blockade of continuous neurogenesis (Sakamoto et al., 2011). These results indicate that continuous neurogenesis is essential for pheromone-associated innately-programmed behaviors and activities. However, conflicting results were also reported. Disruption of neurogenesis in the OB by $\gamma$-ray irradiation left sexual and maternal behaviors unaffected (Feierstein et al., 2010). The discrepancy between these studies might derive from different models and target specificity as described above. One possibility is that newly born neurons in the DG might be involved in such behaviors. Moreover, because current available methods ablate new neurons in both the main and accessory olfactory bulb, it is difficult to conclude which is important for these pheromone-associated behaviors. More restricted ablating method will be required to address these questions.

Although the majority of newly born neurons are incorporated into the main olfactory bulb (MOB), a small number of new neurons migrate into the accessory olfactory bulb (AOB) (Oboti et al., 2009, 2011; Sakamoto et al., 2011). Genetic ablation of newly born neurons revealed that continuous neurogenesis is required for the maintenance of neuronal circuits in the $\mathrm{AOB}$, as observed in the MOB (Sakamoto et al., 2011). However, unlike the MOB, adult neurogenesis does not lead to substantial replacement of GCs in the AOB. This result highlighted a unique integration mode of new neurons in the AOB, suggesting that intrinsic cellular and molecular properties of GCs may be different between the AOB and MOB. Further studies are necessary to elucidate cellular and molecular mechanisms underlying distinct features of GCs in the AOB.

\section{OPTICAL IMAGING AND MANIPULATION OF NEW NEURONS}

Odor information processing is influenced by the activity of $\mathrm{OB}$ interneurons, including pre-existing neurons and newly born neurons. As mentioned above, neurogenesis contributes to various olfaction-related behaviors. However, how new neurons 
contribute to such behaviors is still unclear. New technologies are required to monitor and manipulate the activity of new neurons during such behaviors. Neuronal imaging technologies can help to tackle this issue. During the past decade, two-photon microscope has become a key tool for monitoring the structure, function, and plasticity of neurons in vivo. Calcium imaging is widely used to image the activity of many neurons simultaneously (Grienberger and Konnerth, 2012). In addition, two-photon calcium imaging can monitor the activity of OB GCs in the headfixed awake state (Kato et al., 2012). However, calcium imaging operates too slowly to track the rapid firing of neurons and is also unable to measure the inhibitory signals. An alternative technique, voltage imaging, has a potential to overcome these problems and may ultimately enable to monitor spatiotemporal activity patterns with millisecond order (Peterka et al., 2011). Various kinds of genetically encoded voltage indicators have been developed (Knopfel, 2012). Recently, Akemann and his colleagues succeeded in voltage imaging at a single cell resolution with twophoton microscope in vivo (Akemann et al., 2013). It would be interesting to apply these technologies to examine physiological functions of newly born neurons in the OB. Because most OB newborn neurons are inhibitory interneurons, voltage imaging of $\mathrm{M} / \mathrm{T}$ cells may help to clarify the contribution of GCs in olfactory circuitry more precisely than calcium imaging.

Optogenetics is also a powerful tool in the field of OB neurogenesis. Over the last decade, a wide variety of different kinds of opsins have been developed and become available, and now optogenetic approach is a standard methodology for investigating the functional properties of neurons at the circuit and behavioral level (Fenno et al., 2011). Recently, it was reported that the activation of newly born neurons by channelrhodopsin can accelerate difficult odor discrimination learning and improved odor-associated memory (Alonso et al., 2012). This strategy may also be useful to examine how newly born neurons contribute to pheromoneassociated behaviors. Furthermore, optogenetic tools can control centrifugal input from the olfactory cortex to the OB (Boyd et al., 2012; Markopoulos et al., 2012). It will be interesting to examine how the top-down input affects the survival of new neurons and the effect of odor-associated learning (Yamaguchi et al., 2013). It would be also useful to express optogenetic probes in neurons under activity-dependent control. This approach can allow the reactivation or inactivation of only the subset of neurons that had been activated during a training phase and identify minimal ensemble that are required for behaviors. Light-reactivation of hippocampal neurons that are activated during the training can recall the fear memory of training task (Liu et al., 2012). Because new neurons express immediate-early genes in response to odor stimulation, this approach might be able to identify and manipulate newly born neurons that have been activated by odor stimulation (Magavi et al., 2005).

Although neurogenesis continues throughout life, newly generated neurons dramatically decrease in number with age, and this decline may be involved in memory deficit (Seki and Arai, 1995; Cameron and McKay, 1999; Encinas et al., 2011). In addition, aged mice are impaired at fine olfactory discrimination (Enwere et al., 2004). Furthermore, neurodegenerative diseases are relevant to $\mathrm{OB}$ function and adult neurogenesis. For instance, olfactory dysfunction is well known as an early symptom in Parkinson's disease although there is no specific change in the olfactory epithelium (Braak et al., 2003; Haehner et al., 2009). In Parkinson's disease model ( $\alpha$-synuclein overexpressing mice), the ability of odor discrimination is impaired and the survival of adult born neurons is reduced (Neuner et al., 2014). The next key challenge is to increase neurogenesis in aged/neurodegenerative brain and restore brain functions. Light-sensitive promoter system has a strong potential to achieve it (Wang et al., 2012; Imayoshi et al., 2013; Imayoshi and Kageyama, 2014). This system can control gene expression by blue-light illumination with reversibility. By applying this method in vivo, it might be possible to promote adult neurogenesis even in aged brains and lead to restore brain functions.

\section{CONCLUSION}

Olfaction is indispensable in mammalian life. GCs are the most common GABAergic inhibitory neurons in the OB and modulate the activity of $\mathrm{M} / \mathrm{T}$ cells to shape odor representations. The OB neural circuits are reorganized by incorporation and elimination of newly generated granule neurons throughout life. Furthermore, blockade of neurogenesis results in various olfaction-related behavior defects. Therefore, continuous neurogenesis is important to acquire plasticity in the olfactory system and thereby adapt neural circuits to environmental changes. However, there are still a lot of problems about adult neurogenesis to be solved. For example, molecular mechanisms integrating new neurons into the $\mathrm{OB}$ neural circuits is still unclear. In addition, there are some discrepancies about behavioral analyses. More sophisticated animal model and standardized behavior paradigms should be established. Further studies will contribute to solution of these problems and lead to the development of therapies and drugs for treatment of neurodegenerative diseases.

\section{ACKNOWLEDGMENTS}

Some works reviewed here were conducted in collaboration with M. Yamaguchi and K. Mori at the University of Tokyo. This work was supported by research grants from the Ministry of Education, Culture, Sports, Science, and the Technology of Japan and Japan Science and Technology Agency (JST) as well as Research Fellowships of the Japan Society for the Promotion of Science for Young Scientists (Masayuki Sakamoto), Young Investigator Grants of the Human Frontier Science Program (Itaru Imayoshi), and the JST PRESTO program (Itaru Imayoshi).

\section{REFERENCES}

Adam, Y., and Mizrahi, A. (2011). Long-term imaging reveals dynamic changes in the neuronal composition of the glomerular layer. J. Neurosci. 31, 7967-7973. doi: 10.1523/JNEUROSCI.0782-11.2011

Aimone, J. B., Deng, W., and Gage, F. H. (2011). Resolving new memories: a critical look at the dentate gyrus, adult neurogenesis, and pattern separation. Neuron 70, 589-596. doi: 10.1016/j.neuron.2011.05.010

Akemann, W., Sasaki, M., Mutoh, H., Imamura, T., Honkura, N., and Knopfel, T. (2013). Two-photon voltage imaging using a genetically encoded voltage indicator. Sci. Rep. 3:2231. doi: 10.1038/srep02231

Alonso, M., Lepousez, G., Sebastien, W., Bardy, C., Gabellec, M. M., Torquet, N., et al. (2012). Activation of adult-born neurons facilitates learning and memory. Nat. Neurosci. 15, 897-904. doi: 10.1038/nn.3108 
Alonso, M., Viollet, C., Gabellec, M. M., Meas-Yedid, V., Olivo-Marin, J. C., and Lledo, P. M. (2006). Olfactory discrimination learning increases the survival of adult-born neurons in the olfactory bulb. J. Neurosci. 26, 10508-10513. doi: 10.1523/JNEUROSCI.2633-06.2006

Altman, J., and Das, G. D. (1965). Autoradiographic and histological evidence of postnatal hippocampal neurogenesis in rats. J. Comp. Neurol. 124, 319-335. doi: $10.1002 / \mathrm{cne} .901240303$

Arenkiel, B. R., Hasegawa, H., Yi, J. J., Larsen, R. S., Wallace, M. L., Philpot, B. D., et al. (2011). Activity-induced remodeling of olfactory bulb microcircuits revealed by monosynaptic tracing. PLoS ONE 6:e29423. doi: 10.1371/journal.pone.0029423

Bartolini, G., Ciceri, G., and Marin, O. (2013). Integration of GABAergic interneurons into cortical cell assemblies: lessons from embryos and adults. Neuron 79, 849-864. doi: 10.1016/j.neuron.2013.08.014

Bath, K. G., Mandairon, N., Jing, D., Rajagopal, R., Kapoor, R., Chen, Z. Y., et al. (2008). Variant brain-derived neurotrophic factor (Val66Met) alters adult olfactory bulb neurogenesis and spontaneous olfactory discrimination. J. Neurosci. 28, 2383-2393. doi: 10.1523/JNEUROSCI.4387-07.2008

Batista-Brito, R., Close, J., Machold, R., and Fishell, G. (2008). The distinct temporal origins of olfactory bulb interneuron subtypes. J. Neurosci. 28, 3966-3975. doi: 10.1523/JNEUROSCI.5625-07.2008

Bergmann, O., Liebl, J., Bernard, S., Alkass, K., Yeung, M. S., Steier, P., et al. (2012). The age of olfactory bulb neurons in humans. Neuron 74, 634-639. doi: 10.1016/j.neuron.2012.03.030

Boyd, A. M., Sturgill, J. F., Poo, C., and Isaacson, J. S. (2012). Cortical feedback control of olfactory bulb circuits. Neuron 76, 1161-1174. doi: 10.1016/j.neuron.2012.10.020

Braak, H., Del Tredici, K., Rub, U., De Vos, R. A. I., Steur, E. N. H. J., and Braak, E. (2003). Staging of brain pathology related to sporadic Parkinson's disease. Neurobiol. Aging 24, 197-211. doi: 10.1016/S0197-4580(02)00065-9

Breton-Provencher, V., Lemasson, M., Peralta, M. R. 3rd., and Saghatelyan, A. (2009). Interneurons produced in adulthood are required for the normal functioning of the olfactory bulb network and for the execution of selected olfactory behaviors. J. Neurosci. 29, 15245-15257. doi: 10.1523/JNEUROSCI.3606-09.2009

Brill, M. S., Ninkovic, J., Winpenny, E., Hodge, R. D., Ozen, I., Yang, R., et al. (2009). Adult generation of glutamatergic olfactory bulb interneurons. Nat. Neurosci. 12, 1524-1533. doi: 10.1038/nn.2416

Bruce, H. M. (1959). An exteroceptive block to pregnancy in the mouse. Nature 184, 105. doi: 10.1038/184105a0

Cameron, H. A., and McKay, R. D. (1999). Restoring production of hippocampal neurons in old age. Nat. Neurosci. 2, 894-897. doi: 10.1038/13197

Clelland, C. D., Choi, M., Romberg, C., Clemenson, G. D. Jr., Fragniere, A., Tyers, P., et al. (2009). A functional role for adult hippocampal neurogenesis in spatial pattern separation. Science 325, 210-213. doi: 10.1126/science.1173215

Deng, W., Aimone, J. B., and Gage, F. H. (2010). New neurons and new memories: how does adult hippocampal neurogenesis affect learning and memory? Nat. Rev. Neurosci. 11, 339-350. doi: 10.1038/nrn2822

Deshpande, A., Bergami, M., Ghanem, A., Conzelmann, K. K., Lepier, A., Gotz, M. et al. (2013). Retrograde monosynaptic tracing reveals the temporal evolution of inputs onto new neurons in the adult dentate gyrus and olfactory bulb. Proc. Natl. Acad. Sci. U.S.A. 110, 1152-1161. doi: 10.1073/pnas. 1218991110

Egger, V., and Urban, N. N. (2006). Dynamic connectivity in the mitral cell-granule cell microcircuit. Semin. Cell Dev. Biol. 17, 424-432. doi: 10.1016/j.semcdb.2006.04.006

Encinas, J. M., Michurina, T. V., Peunova, N., Park, J. H., Tordo, J., Peterson, D. A., et al. (2011). Division-coupled astrocytic differentiation and age-related depletion of neural stem cells in the adult hippocampus. Cell Stem Cell 8, 566-579. doi: 10.1016/j.stem.2011.03.010

Enwere, E., Shingo, T., Gregg, C., Fujikawa, H., Ohta, S., and Weiss, S. (2004). Aging results in reduced epidermal growth factor receptor signaling, diminished olfactory neurogenesis, and deficits in fine olfactory discrimination. J. Neurosci. 24 8354-8365. doi: 10.1523/JNEUROSCI.2751-04.2004

Eriksson, P. S., Perfilieva, E., Bjork-Eriksson, T., Alborn, A. M., Nordborg, C. Peterson, D. A., et al. (1998). Neurogenesis in the adult human hippocampus. Nat. Med. 4, 1313-1317. doi: 10.1038/3305

Ernst, A., Alkass, K., Bernard, S., Salehpour, M., Perl, S., Tisdale, J., et al. (2014). Neurogenesis in the striatum of the adult human brain. Cell 156, 1072-1083. doi: 10.1016/j.cell.2014.01.044
Eyre, M. D., Antal, M., and Nusser, Z. (2008). Distinct deep shortaxon cell subtypes of the main olfactory bulb provide novel intrabulbar and extrabulbar GABAergic connections. J. Neurosci. 28, 8217-8229. doi: 10.1523/JNEUROSCI.2490-08.2008

Eyre, M. D., Kerti, K., and Nusser, Z. (2009). Molecular diversity of deep shortaxon cells of the rat main olfactory bulb. Eur. J. Neurosci. 29, 1397-1407. doi: 10.1111/j.1460-9568.2009.06703.x

Feierstein, C. E., Lazarini, F., Wagner, S., Gabellec, M. M., De Chaumont, F., OlivoMarin, J. C., et al. (2010). Disruption of adult neurogenesis in the olfactory bulb affects social interaction but not maternal behavior. Front. Behav. Neurosci. 4:176. doi: 10.3389/fnbeh.2010.00176

Fenno, L., Yizhar, O., and Deisseroth, K. (2011). The development and application of optogenetics. Annu. Rev. Neurosci. 34, 389-412. doi: 10.1146/annurev-neuro061010-113817

Fuentealba, L. C., Obernier, K., and Alvarez-Buylla, A. (2012). Adult neural stem cells bridge their niche. Cell Stem Cell 10, 698-708. doi: 10.1016/j.stem.2012.05.012

Gheusi, G., Cremer, H., McLean, H., Chazal, G., Vincent, J. D., and Lledo, P. M. (2000). Importance of newly generated neurons in the adult olfactory bulb for odor discrimination. Proc. Natl. Acad. Sci. U.S.A. 97, 1823-1828. doi: 10.1073/pnas.97.4.1823

Ghosh, S., Larson, S. D., Hefzi, H., Marnoy, Z., Cutforth, T., Dokka, K., et al. (2011). Sensory maps in the olfactory cortex defined by long-range viral tracing of single neurons. Nature 472, 217-220. doi: 10.1038/nature09945

Goldman, S. A., and Nottebohm, F. (1983). Neuronal production, migration, and differentiation in a vocal control nucleus of the adult female canary brain. Proc. Natl. Acad. Sci. U.S.A. 80, 2390-2394. doi: 10.1073/pnas.80.8.2390

Grienberger, C., and Konnerth, A. (2012). Imaging calcium in neurons. Neuron 73, 862-885. doi: 10.1016/j.neuron.2012.02.011

Haehner, A., Hummel, T., and Reichmann, H. (2009). Olfactory dysfunction as a diagnostic marker for Parkinson's disease. Expert Rev. Neurother. 9, 1773-1779. doi: 10.1586/ern.09.115

Huang, L., Garcia, I., Jen, H. I., and Arenkiel, B. R. (2013). Reciprocal connectivity between mitral cells and external plexiform layer interneurons in the mouse olfactory bulb. Front. Neural Circuits 7:32. doi: 10.3389/fncir.2013.00032

Huang, Z. J., and Zeng, H. (2013). Genetic approaches to neural circuits in the mouse. Annu. Rev. Neurosci. 36, 183-215. doi: 10.1146/annurev-neuro-062012170307

Igarashi, K. M., Ieki, N., An, M., Yamaguchi, Y., Nagayama, S., Kobayakawa, K., et al. (2012). Parallel mitral and tufted cell pathways route distinct odor information to different targets in the olfactory cortex. J. Neurosci. 32, 7970-7985. doi: 10.1523/JNEUROSCI.0154-12.2012

Imamura, F., Ayoub, A. E., Rakic, P., and Greer, C. A. (2011). Timing of neurogenesis is a determinant of olfactory circuitry. Nat. Neurosci. 14, 331-337. doi: $10.1038 / \mathrm{nn} .2754$

Imamura, F., Nagao, H., Naritsuka, H., Murata, Y., Taniguchi, H., and Mori, K. (2006). A leucine-rich repeat membrane protein, 5T4, is expressed by a subtype of granule cells with dendritic arbors in specific strata of the mouse olfactory bulb. J. Comp. Neurol. 495, 754-768. doi: 10.1002/cne.20896

Imayoshi, I., Isomura, A., Harima, Y., Kawaguchi, K., Kori, H., Miyachi, H., et al. (2013). Oscillatory control of factors determining multipotency and fate in mouse neural progenitors. Science 342, 1203-1208. doi: 10.1126/science. 1242366

Imayoshi, I., and Kageyama, R. (2014). bHLH factors in self-renewal, multipotency, and fate choice of neural progenitor cells. Neuron 82, 9-23. doi: 10.1016/j.neuron.2014.03.018

Imayoshi, I., Sakamoto, M., and Kageyama, R. (2011). Genetic methods to identify and manipulate newly born neurons in the adult brain. Front. Neurosci. 5:64. doi: 10.3389/fnins.2011.00064

Imayoshi, I., Sakamoto, M., Ohtsuka, T., Takao, K., Miyakawa, T., Yamaguchi, M., et al. (2008). Roles of continuous neurogenesis in the structural and functional integrity of the adult forebrain. Nat. Neurosci. 11, 1153-1161. doi: 10.1038/nn.2185

Isaacson, J. S., and Strowbridge, B. W. (1998). Olfactory reciprocal synapses: dendritic signaling in the CNS. Neuron 20, 749-761. doi: 10.1016/S08966273(00)81013-2

Kaplan, M. S., and Hinds, J. W. (1977). Neurogenesis in the adult rat: electron microscopic analysis of light radioautographs. Science 197, 1092-1094. doi: $10.1126 /$ science. 887941 
Kato, H. K., Chu, M. W., Isaacson, J. S., and Komiyama, T. (2012). Dynamic sensory representations in the olfactory bulb: modulation by wakefulness and experience. Neuron 76, 962-975. doi: 10.1016/j.neuron.2012.09.037

Kato, H. K., Gillet, S. N., Peters, A. J., Isaacson, J. S., and Komiyama, T. (2013). Parvalbumin-expressing interneurons linearly control olfactory bulb output. Neuron 80, 1218-1231. doi: 10.1016/j.neuron.2013.08.036

Kelsch, W., Lin, C. W., and Lois, C. (2008). Sequential development of synapses in dendritic domains during adult neurogenesis. Proc. Natl. Acad. Sci. U.S.A. 105, 16803-16808. doi: 10.1073/pnas.0807970105

Kelsch, W., Sim, S., and Lois, C. (2010). Watching synaptogenesis in the adult brain. Annu. Rev. Neurosci. 33, 131-149. doi: 10.1146/annurev-neuro-060909-153252

Kim, W. R., Kim, Y., Eun, B., Park, O. H., Kim, H., Kim, K., et al. (2007). Impaired migration in the rostral migratory stream but spared olfactory function after the elimination of programmed cell death in Bax knock-out mice. J. Neurosci. 27, 14392-14403. doi: 10.1523/JNEUROSCI.3903-07.2007

Knopfel, T. (2012). Genetically encoded optical indicators for the analysis of neuronal circuits. Nat. Rev. Neurosci. 13, 687-700. doi: 10.1038/nrn3293

Kopel, H., Schechtman, E., Groysman, M., and Mizrahi, A. (2012). Enhanced synaptic integration of adult-born neurons in the olfactory bulb of lactating mothers. J. Neurosci. 32, 7519-7527. doi: 10.1523/JNEUROSCI.6354-11.2012

Kosaka, K., Toida, K., Aika, Y., and Kosaka, T. (1998). How simple is the organization of the olfactory glomerulus?: the heterogeneity of so-called periglomerular cells. Neurosci. Res. 30, 101-110. doi: 10.1016/S0168-0102(98)00002-9

Kosaka, T., and Kosaka, K. (2011). "Interneurons" in the olfactory bulb revisited. Neurosci. Res. 69, 93-99. doi: 10.1016/j.neures.2010.10.002

Kriegstein, A., and Alvarez-Buylla, A. (2009). The glial nature of embryonic and adult neural stem cells. Annu. Rev. Neurosci. 32, 149-184. doi: 10.1146/annurev.neuro.051508.135600

Larsen, C. M., and Grattan, D. R. (2010). Prolactin-induced mitogenesis in the subventricular zone of the maternal brain during early pregnancy is essential for normal postpartum behavioral responses in the mother. Endocrinology 151, 3805-3814. doi: 10.1210/en.2009-1385

Lazarini, F., Mouthon, M. A., Gheusi, G., De Chaumont, F., Olivo-Marin, J. C., Lamarque, S., et al. (2009). Cellular and behavioral effects of cranial irradiation of the subventricular zone in adult mice. PLoS ONE 4:e7017. doi 10.1371/journal.pone.0007017

Lemasson, M., Saghatelyan, A., Olivo-Marin, J. C., and Lledo, P. M. (2005). Neonatal and adult neurogenesis provide two distinct populations of newborn neurons to the mouse olfactory bulb. J. Neurosci. 25, 6816-6825. doi: 10.1523/JNEUROSCI.1114-05.2005

Lepousez, G., and Lledo, P. M. (2013). Odor discrimination requires proper olfactory fast oscillations in awake mice. Neuron 80, 1010-1024. doi: 10.1016/j.neuron.2013.07.025

Lepousez, G., Valley, M. T., and Lledo, P. M. (2013). The impact of adult neurogenesis on olfactory bulb circuits and computations. Annu. Rev. Physiol. 75, 339-363. doi: 10.1146/annurev-physiol-030212-183731

Liu, X., Ramirez, S., Pang, P. T., Puryear, C. B., Govindarajan, A., Deisseroth, K., et al. (2012). Optogenetic stimulation of a hippocampal engram activates fear memory recall. Nature 484, 381-385. doi: 10.1038/nature11028

Livneh, Y., Adam, Y., and Mizrahi, A. (2014). Odor processing by adult-born neurons. Neuron 81, 1097-1110. doi: 10.1016/j.neuron.2014.01.007

Livneh, Y., and Mizrahi, A. (2012). Experience-dependent plasticity of mature adult-born neurons. Nat. Neurosci. 15, 26-28. doi: 10.1038/nn.2980

Lledo, P. M., Alonso, M., and Grubb, M. S. (2006). Adult neurogenesis and functional plasticity in neuronal circuits. Nat. Rev. Neurosci. 7, 179-193. doi: $10.1038 / \mathrm{nrn} 1867$

Magavi, S. S., Mitchell, B. D., Szentirmai, O., Carter, B. S., and Macklis, J. D. (2005). Adult-born and preexisting olfactory granule neurons undergo distinct experience-dependent modifications of their olfactory responses in vivo. J. Neurosci. 25, 10729-10739. doi: 10.1523/JNEUROSCI.2250-05.2005

Mak, G. K., Enwere, E. K., Gregg, C., Pakarainen, T., Poutanen, M., Huhtaniemi, I., et al. (2007). Male pheromone-stimulated neurogenesis in the adult female brain: possible role in mating behavior. Nat. Neurosci. 10, 1003-1011. doi: 10.1038/nn1928

Mak, G. K., and Weiss, S. (2010). Paternal recognition of adult offspring mediated by newly generated CNS neurons. Nat. Neurosci. 13, 753-758. doi: $10.1038 / \mathrm{nn} .2550$

Manabe, H., Kusumoto-Yoshida, I., Ota, M., and Mori, K. (2011). Olfactory cortex generates synchronized top-down inputs to the olfactory bulb during slow-wave sleep. J. Neurosci. 31, 8123-8133. doi: 10.1523/JNEUROSCI.657810.2011

Markopoulos, F., Rokni, D., Gire, D. H., and Murthy, V. N. (2012). Functional properties of cortical feedback projections to the olfactory bulb. Neuron 76, 1175-1188. doi: 10.1016/j.neuron.2012.10.028

Ming, G. L., and Song, H. (2011). Adult neurogenesis in the mammalian brain: significant answers and significant questions. Neuron 70, 687-702. doi: 10.1016/j.neuron.2011.05.001

Miyamichi, K., Amat, F., Moussavi, F., Wang, C., Wickersham, I., Wall, N. R., et al. (2011). Cortical representations of olfactory input by trans-synaptic tracing. Nature 472, 191-196. doi: 10.1038/nature09714

Miyamichi, K., Shlomai-Fuchs, Y., Shu, M., Weissbourd, B. C., Luo, L., and Mizrahi, A. (2013). Dissecting local circuits: parvalbumin interneurons underlie broad feedback control of olfactory bulb output. Neuron 80, 1232-1245. doi: 10.1016/j.neuron.2013.08.027

Moreno, M. M., Linster, C., Escanilla, O., Sacquet, J., Didier, A., and Mandairon, N. (2009). Olfactory perceptual learning requires adult neurogenesis. Proc. Natl. Acad. Sci. U.S.A. 106, 17980-17985. doi: 10.1073/pnas.0907 063106

Mori, K., Kishi, K., and Ojima, H. (1983). Distribution of dendrites of mitral, displaced mitral, tufted, and granule cells in the rabbit olfactory bulb. J. Comp. Neurol. 219, 339-355. doi: 10.1002/cne.902190308

Mori, K., and Sakano, H. (2011). How is the olfactory map formed and interpreted in the mammalian brain? Annu. Rev. Neurosci. 34, 467-499. doi: 10.1146/annurev-neuro-112210-112917

Mouret, A., Lepousez, G., Gras, J., Gabellec, M. M., and Lledo, P. M. (2009). Turnover of newborn olfactory bulb neurons optimizes olfaction. J. Neurosci. 29, 12302-12314. doi: 10.1523/JNEUROSCI.3383-09.2009

Nakashiba, T., Cushman, J. D., Pelkey, K. A., Renaudineau, S., Buhl, D. L., McHugh, T. J., et al. (2012). Young dentate granule cells mediate pattern separation, whereas old granule cells facilitate pattern completion. Cell 149, 188-201. doi: 10.1016/j.cell.2012.01.046

Neuner, J., Filser, S., Michalakis, S., Biel, M., and Herms, J. (2014). A30P alphaSynuclein interferes with the stable integration of adult-born neurons into the olfactory network. Sci. Rep. 4:3931. doi: 10.1038/srep03931

Ninkovic, J., Mori, T., and Gotz, M. (2007). Distinct modes of neuron addition in adult mouse neurogenesis. J. Neurosci. 27, 10906-10911. doi: 10.1523/JNEUROSCI.2572-07.2007

Nissant, A., Bardy, C., Katagiri, H., Murray, K., and Lledo, P. M. (2009). Adult neurogenesis promotes synaptic plasticity in the olfactory bulb. Nat. Neurosci. 12, 728-730. doi: 10.1038/nn.2298

Nunez-Parra, A., Maurer, R. K., Krahe, K., Smith, R. S., and Araneda, R. C. (2013). Disruption of centrifugal inhibition to olfactory bulb granule cells impairs olfactory discrimination. Proc. Natl. Acad. Sci. U.S.A. 110, 14777-14782. doi: 10.1073/pnas. 1310686110

Nunez-Parra, A., Pugh, V., and Araneda, R. C. (2011). Regulation of adult neurogenesis by behavior and age in the accessory olfactory bulb. Mol. Cell. Neurosci. 47, 274-285. doi: 10.1016/j.mcn.2011.05.003

Oboti, L., Savalli, G., Giachino, C., De Marchis, S., Panzica, G. C., Fasolo, A. et al. (2009). Integration and sensory experience-dependent survival of newlygenerated neurons in the accessory olfactory bulb of female mice. Eur. J. Neurosci. 29, 679-692. doi: 10.1111/j.1460-9568.2009.06614.x

Oboti, L., Schellino, R., Giachino, C., Chamero, P., Pyrski, M., LeindersZufall, T., et al. (2011). Newborn interneurons in the accessory olfactory bulb promote mate recognition in female mice. Front. Neurosci. 5:113. doi: 10.3389/fnins.2011.00113

Orona, E., Scott, J. W., and Rainer, E. C. (1983). Different granule cell populations innervate superficial and deep regions of the external plexiform layer in rat olfactory bulb. J. Comp. Neurol. 217, 227-237. doi: 10.1002/cne.902170209

Peterka, D. S., Takahashi, H., and Yuste, R. (2011). Imaging voltage in neurons. Neuron 69, 9-21. doi: 10.1016/j.neuron.2010.12.010

Petreanu, L., and Alvarez-Buylla, A. (2002). Maturation and death of adultborn olfactory bulb granule neurons: role of olfaction. J. Neurosci. 22, 6106-6113.

Pressler, R. T., and Strowbridge, B. W. (2006). Blanes cells mediate persistent feedforward inhibition onto granule cells in the olfactory bulb. Neuron 49, 889-904. doi: 10.1016/j.neuron.2006.02.019

Ramon y Cajal, S. (1928). Degeneration and Regeneration of the Nervous System. London: Oxford University Press. 
Reynolds, B. A., and Weiss, S. (1992). Generation of neurons and astrocytes from isolated cells of the adult mammalian central nervous system. Science 255, 1707-1710. doi: 10.1126/science. 1553558

Rochefort, C., Gheusi, G., Vincent, J. D., and Lledo, P. M. (2002). Enriched odor exposure increases the number of newborn neurons in the adult olfactory bulb and improves odor memory. J. Neurosci. 22, 2679-2689.

Sahay, A., Scobie, K. N., Hill, A. S., O’Carroll, C. M., Kheirbek, M. A., Burghardt, N. S., et al. (2011a). Increasing adult hippocampal neurogenesis is sufficient to improve pattern separation. Nature 472, 466-470. doi: 10.1038/nature09817

Sahay, A., Wilson, D. A., and Hen, R. (2011b). Pattern separation: a common function for new neurons in hippocampus and olfactory bulb. Neuron 70, 582-588. doi: 10.1016/j.neuron.2011.05.012

Sakamoto, M., Ieki, N., Miyoshi, G., Mochimaru, D., Miyachi, H., Imura, T., et al. (2014). Continuous postnatal neurogenesis contributes to formation of the olfactory bulb neural circuits and flexible olfactory associative learning. J. Neurosci. 34, 5788-5799. doi: 10.1523/JNEUROSCI.0674-14.2014

Sakamoto, M., Imayoshi, I., Ohtsuka, T., Yamaguchi, M., Mori, K., and Kageyama, R. (2011). Continuous neurogenesis in the adult forebrain is required for innate olfactory responses. Proc. Natl. Acad. Sci. U.S.A. 108, 8479-8484. doi: 10.1073/pnas. 1018782108

Sanai, N., Nguyen, T., Ihrie, R. A., Mirzadeh, Z., Tsai, H. H., Wong, M., et al. (2011). Corridors of migrating neurons in the human brain and their decline during infancy. Nature 478, 382-386. doi: 10.1038/nature10487

Sawada, M., Kaneko, N., Inada, H., Wake, H., Kato, Y., Yanagawa, Y., et al. (2011). Sensory input regulates spatial and subtype-specific patterns of neuronal turnover in the adult olfactory bulb. J. Neurosci. 31, 11587-11596. doi: 10.1523/JNEUROSCI.0614-11.2011

Schellinck, H. M., Arnold, A., and Rafuse, V. F. (2004). Neural cell adhesion molecule (NCAM) null mice do not show a deficit in odour discrimination learning. Behav. Brain Res. 152, 327-334. doi: 10.1016/j.bbr.2003.10.011

Seki, T., and Arai, Y. (1995). Age-related production of new granule cells in the adult dentate gyrus. Neuroreport 6, 2479-2482. doi: 10.1097/00001756-1995121 50-00010

Shepherd, G., and Greer, C. (2004). Olfactory Bulb in the Synaptic Organization of the Brain. New York, NY: Oxford University Press. doi: 10.1093/acprof:oso/ 9780195159561.001.1

Shingo, T., Gregg, C., Enwere, E., Fujikawa, H., Hassam, R., Geary, C., et al. (2003). Pregnancy-stimulated neurogenesis in the adult female forebrain mediated by prolactin. Science 299, 117-120. doi: 10.1126/science.1076647

Soria-Gomez, E., Bellocchio, L., Reguero, L., Lepousez, G., Martin, C., Bendahmane, M., et al. (2014). The endocannabinoid system controls food intake via olfactory processes. Nat. Neurosci. 17, 407-415. doi: 10.1038/nn.3647

Sosulski, D. L., Bloom, M. L., Cutforth, T., Axel, R., and Datta, S. R. (2011). Distinct representations of olfactory information in different cortical centres. Nature 472, 213-216. doi: 10.1038/nature09868
Spalding, K. L., Bergmann, O., Alkass, K., Bernard, S., Salehpour, M., Huttner, H. B., et al. (2013). Dynamics of hippocampal neurogenesis in adult humans. Cell 153, 1219-1227. doi: 10.1016/j.cell.2013.05.002

Suh, H., Deng, W., and Gage, F. H. (2009). Signaling in adult neurogenesis. Annu. Rev. Cell Dev. Biol. 25, 253-275. doi: 10.1146/annurev.cellbio.042308.113256

Sultan, S., Mandairon, N., Kermen, F., Garcia, S., Sacquet, J., and Didier, A. (2010). Learning-dependent neurogenesis in the olfactory bulb determines long-term olfactory memory. FASEB J. 24, 2355-2363. doi: 10.1096/fj.09-151456

Sultan, S., Rey, N., Sacquet, J., Mandairon, N., and Didier, A. (2011). Newborn neurons in the olfactory bulb selected for long-term survival through olfactory learning are prematurely suppressed when the olfactory memory is erased. J. Neurosci. 31, 14893-14898. doi: 10.1523/JNEUROSCI.3677-11.2011

Wang, X., Chen, X., and Yang, Y. (2012). Spatiotemporal control of gene expression by a light-switchable transgene system. Nat. Methods 9, 266-269. doi: 10.1038/nmeth.1892

Yamaguchi, M., Manabe, H., Murata, K., and Mori, K. (2013). Reorganization of neuronal circuits of the central olfactory system during postprandial sleep. Front. Neural Circuits 7:132. doi: 10.3389/fncir.2013.00132

Yamaguchi, M., and Mori, K. (2005). Critical period for sensory experiencedependent survival of newly generated granule cells in the adult mouse olfactory bulb. Proc. Natl. Acad. Sci. U.S.A. 102, 9697-9702. doi: 10.1073/pnas. 0406082102

Yokoi, M., Mori, K., and Nakanishi, S. (1995). Refinement of odor molecule tuning by dendrodendritic synaptic inhibition in the olfactory bulb. Proc. Natl. Acad. Sci. U.S.A. 92, 3371-3375. doi: 10.1073/pnas.92.8.3371

Yokoyama, T. K., Mochimaru, D., Murata, K., Manabe, H., Kobayakawa, K., Kobayakawa, R., et al. (2011). Elimination of adult-born neurons in the olfactory bulb is promoted during the postprandial period. Neuron 71, 883-897. doi: 10.1016/j.neuron.2011.05.046

Conflict of Interest Statement: The authors declare that the research was conducted in the absence of any commercial or financial relationships that could be construed as a potential conflict of interest.

Received: 24 February 2014; accepted: 06 May 2014; published online: 26 May 2014. Citation: Sakamoto M, Kageyama R and Imayoshi I (2014) The functional significance of newly born neurons integrated into olfactory bulb circuits. Front. Neurosci. 8:121. doi: $10.3389 /$ fnins.2014.00121

This article was submitted to Neurogenesis, a section of the journal Frontiers in Neuroscience.

Copyright (C) 2014 Sakamoto, Kageyama and Imayoshi. This is an open-access article distributed under the terms of the Creative Commons Attribution License (CC BY). The use, distribution or reproduction in other forums is permitted, provided the original author(s) or licensor are credited and that the original publication in this journal is cited, in accordance with accepted academic practice. No use, distribution or reproduction is permitted which does not comply with these terms. 